\section{These short summaries are taken from a range of other evidence-based journals and publications}

\author{
Derek Richards
}

Centre for Evidence-based Dentistry, Oxford, UK

\section{No evidence to show that acupuncture is an effective treatment for temporomandibular joint dysfunction}

\author{
Electronic Bandolier, \\ (www.jr2.ox.ac.uk/Bandolier/booth/alternat/AT005.html)
}

Ernst E, White AR. Acupuncture as a treatment for temporomandibular joint dysfunction: a systematic review of randomised trials. Arch Otolaryngol Head Neck Surg 1999; 125:269-272.

This 1997 systematic review, with extensive searches of Medline, EMBASE, CISCOM, the Cochrane Library and reference lists, retrieved three randomised-controlled trials and a total of 205 patients. Inclusion criteria were RCT of acupuncture compared with sham acupuncture, standard treatment or no treatment. Patients in all studies were hospital specialist referrals from family dentists or doctors for temporomandibular joint dysfunction treatment.

All three trials had serious methodological flaws; none of the trials had an adequate placebo control group. The fact that acupuncture was better than no treatment does not rule out a placebo effect. In these trials, acupuncture was shown to be as effective as standard treatment. The trials were not sensitive for the long-term outcomes however, and, in the absence of a placebo group or an additional active control group of proven efficacy, it is unclear if both treatments were indistinguishable from each other because they were equally effective or equally ineffective. The purported long-term effects of acupuncture and standard therapy may be merely due to the natural history of the disease. These trials are inadequately designed to answer these questions.

\section{Acupuncture trials and quality}

\section{Electronic Bandolier,} (www.jr2.ox.ac.uk/Bandolier/band80/b80-3.html)

Ezzo J, Berman B, Hadhazy VA, Jadad AR, Lao L, Singh BB. Is acupuncture effective for the treatment of chronic pain? A systematic review. Pain 2000; Jun;86(3):217-225.
Acupuncture is commonly believed to be effective for the treatment of chronic pain, despite growing evidence from systematic reviews that it is not. This systematic review across all areas of chronic pain draws attention to the fact that the number of clinical trials of high quality that showed acupuncture to be effective is three.

The search strategy was extensive and included standard databases and at least four specialist databases for complementary therapies, as well as dissertation abstracts and conference proceedings. There were 50 trials with 2394 patients; $68 \%$ of the trials had a quality score of 2 or less on a five-point scale. Controls included waiting lists, inert controls, sham acupuncture and active controls, usually transcutaneous electrical nerve stimulation.

$40-50 \%$ of the trials or patients showed acupuncture to be better than control treatments. The high-quality studies either showed no benefit or that acupuncture was worse than control, however, and lower-quality studies showed significantly higher treatment effect than those of high quality.

Acupuncture is much used for chronic pain and yet there is an absence of significant effect. Many of the studies were small. Of the 19 positive studies, 14 enrolled fewer than 50 patients with 12 the smallest number. Overall, positive studies were smaller than neutral studies, which were smaller than negative studies. We might conclude that there is some residual bias in this, which would result in an even more negative conclusion.

This emphasises that consumers and providers should beware: this review shows that the use of acupuncture for chronic pain is not supported by any evidence of quality.

\section{Early treatment of acute herpes zoster may prevent or shorten the duration of post herpetic neuralgia}

Evidence-based Medicine, 2000; 5:144

Alper BS, Lewis PR. Does treatment of acute herpes zoster prevent or shorten postherpetic neuralgia? A systematic review of the literature. J Fam Pract 2000; 49:255-264.

This systematic review includes 42 studies including studies from four previous meta-analyses [one of which has been summarised in Evidence-Based Dentistry 1998; 1(1):16]. Recent evidence suggests that post-herpetic neuralgia (PHN) is less common that previously thought, with fewer than one in 10 patients over 50 years of age having continuing pain at 1 year after infection. Nevertheless, the distress caused by PHN makes an effective preventive treatment worth having. The weight of evidence supports the benefit of 
prescribing antiviral agents for people who have a higher risk for PHN (i.e., those over 50 years of age).

One trial included, however, suggested that amitriptyline may reduce the incidence of $\mathrm{PHN}$ when used during the acute zoster attack, in addition to its benefits in improving established PHN. Tricyclic antidepressants are much less costly than antivirals [see Evidence-Based Dentistry 1999; 1(2):30]. Unfortunately, as this trial allowed co-prescribing of antivirals, there is not enough evidence to justify amitriptyline without antiviral agents as an initial treatment strategy.

\section{Pacifiers have greater analgesic effect than sweet solutions during venipuncture in full term newborns}

Evidence-based Medicine, 2000; 5:122
Carbajal R, Chauvet X, Couderc S, Olivier-Martin M. Randomised trial of analgesic effect of sucrose, glucose and pacifiers in term neonates. BMJ 1999; 319:1393-1397.

This randomised controlled trial involving 150 newborns compared the effect of glucose, sucrose and pacifiers on pain during venipuncture. Pacifiers were found to be more effective than the sweet solutions. The article discusses the possible mechanism for the action of pacifiers, although the investigators did recognise the potential to bias since pacifier use is not blinded. The efficacy of pacifiers in other procedures remains to be determined but, despite the fact that the use of pacifiers is often discouraged, this study shows them to be more effective than $30 \%$ glucose or $30 \%$ sucrose solutions during venipuncture: a finding that dentists will appreciate. 